\title{
ADVERTENCIAS DEL PANEL INTERGUBERNAMENTAL DEL CAMBIO CLIMÁTICO*
}

\section{EL IPCC CONSIDERA QUE EL DECRECIMIENTO ES CLAVE PARA MITIGAR EL CAMBIO CLIMÁTICO}

\author{
Juan Bordera ${ }^{\mathrm{a}}$ \\ Fernando Prieto
}

E1 pasado 23 de junio ocurrió algo muy poco común. AFP (Agencia France Press filtró parte del contenido del resumen para políticos del Grupo II del IPCC (Panel Intergubernamental del Cambio Climático) -el encargado de analizar los impactos del cambio climático-. La noticia dio la vuelta al mundo, y el titular que más se repetía -extraído del propio informe- era: "La vida en la Tierra puede recuperarse de un cambio climático importante evolucionando hacia nuevas especies y creando nuevos ecosistemas. La humanidad no”. La anomalía se ha repetido antes de cumplirse dos meses. Otra brecha en el antaño hermético IPCC, otra filtración.

DOI: https://doi.org/10.18601/01245996.v24n46.12. Recepción: 10-092021, aceptación: 29-11-2021. Sugerencia de citación: Bordera, J., Prieto, F., Valladares, F., Turiel, A. Puig V, F. y Hewlett, T. (2022). Advertencias del Panel Intergubernamental del Cambio Climático. Revista de Economía Institucional, 24(46), 237-247. Tomado de Revista Contexto, 7 y 22-08-2021, [https://ctxt.es/]

a Periodista, guionista y activista en Extinction Rebellion España y València en Transició.

b Doctor en Ecología y director del Observatorio de la Sostenibilidad. 
CTXT ha tenido acceso al contenido de otra parte del Sexto Informe del IPCC, el organismo en el que científicos y científicas de todo el mundo, de diferentes áreas del saber, colaboran voluntariamente y por prestigio, en uno de los esfuerzos de cooperación internacional más esperanzadores que existen. En el IPCC buscan dar forma a una serie de informes que, últimamente cada 5-6 años, actualizan el conocimiento sobre el desafío - con dos caras que se entrelazan- más importante que tenemos: adaptarnos al caos climático que ya hemos generado, y dilucidar cómo transitar hacia una economía y un modelo energético que puedan perdurar en el tiempo. Sus esfuerzos se dividen en tres grupos para elaborar sendos informes que se relacionan entre ellos: Ciencia (Grupo I), Impactos (Grupo II) y Mitigación (Grupo III). Durante ese periodo también se publican informes parciales concretos.

La explicación de por qué están ocurriendo estas filtraciones no admite dudas: hay mucha gente dentro del propio organismo muy preocupada por la situación actual de emergencia, por la tibieza de algunas de las conclusiones de los sucesivos informes, y también por la manifiesta dificultad de plasmar en políticas las medidas propuestas. E1 IPCC lleva funcionando desde 1990, y desde ese momento hasta ahora no ha ocurrido otra cosa que un muy evidente aumento en las emisiones y en los efectos colaterales negativos, aunque esto sea responsabilidad de las inercias económicas, sociales y políticas. Por eso se está filtrando el contenido de los resúmenes para políticos, para evitar que se pueda maquillar mucho el informe en el proceso, y para llamar la atención sobre el mismo en una década en la que nos lo vamos a jugar todo.

¿Cuál es la situación actual? Muy resumidamente: no paran de sucederse récords de temperatura extraordinarios por todas partes, como el de Canadá hace un mes, donde se batió el registro anterior durante tres días consecutivos para llegar a cinco grados más de golpe, muy cerca de los $50^{\circ} \mathrm{C}$. También están ocurriendo inundaciones fuera de toda lógica, como las de Alemania, Bélgica o China, con cientos de desaparecidos y víctimas mortales, y por supuesto enormes daños económicos, además de tremendos incendios en buena parte del planeta. Estos últimos días les ha tocado a Grecia y a Turquía el boleto perdedor en la lotería climática. Una lotería en la que todos los países tienen muchos números y no habrá ningún ganador.

Una vez hechas las presentaciones, pasemos al informe. En este caso la filtración es sobre el primer borrador del resumen para políticos del Grupo III, el encargado de analizar cómo reducir las emisiones, 
mitigar y atenuar los impactos. Algunas de las líneas maestras que podemos extraer del borrador del informe, cuyo contenido definitivo se publicará en marzo de 2022, son:

- "Sería necesario que las emisiones de $\mathrm{CO}_{2}$ lleguen a su máximo antes de 2025 y que lleguen al cero neto entre 2050 y 2075”. Esto implica mayor ambición en el corto y medio plazo, y acelerar las actuaciones y la implementación efectiva, que están chocando con obstáculos políticos, económicos y sociales. Aquello que puede ser más eficiente desde el punto de vista económico, puede ser políticamente inviable o éticamente inaceptable. Y esto es clave, los cambios deben tener muy en cuenta la desigualdad para ser aceptados (véase el caso de los chalecos amarillos).

- "No habría que construir ninguna nueva planta de carbón o gas, y las actuales deberían reducir su vida útil”, que es típicamente de más de 30 años, a alrededor de 10 años.

- Se reconoce que para conseguir el cero neto en las emisiones se necesita un cierto grado de captura y secuestro de carbono y eliminación de carbono (CDR-CCS-BECCS). Unas tecnologías que no están desarrolladas ni mucho menos, y que representan otra patada hacia adelante pensando que la evolución tecnológica vendrá siempre al rescate. Contradiciendo absolutamente uno de los principios básicos de la ciencia: el principio de precaución. Por si esto fuera poco, hay investigaciones que plantean dudas al respecto del potencial del suelo para almacenar tanto carbono. Más aún en un planeta que se calienta.

- "El cambio tecnológico implementado hasta ahora a nivel global no es suficiente para alcanzar los objetivos climáticos ni de desarrollo. Desde 2010 el coste de las tecnologías renovables ha disminuido por encima de las expectativas (especialmente la solar $-87 \%$, y las baterías $-85 \%$ ), pero en total, solar y eólica representan el $7 \%$ del suministro eléctrico". Los avances esperados en otras tecnologías como la mencionada captura y secuestro de carbono, energía nuclear y CDR (eliminación de dióxido de carbono) han sido mucho menos esperanzadores.

- "El crecimiento del consumo de energía y materiales es la causa principal del incremento de Gases de Efecto Invernadero (GEI). E1 ligero desacoplamiento observado del crecimiento respecto al uso de energía [y motivado en gran parte por la deslocalización de la producción] no ha podido contrarrestar el efecto del crecimiento económico y poblacional". Esto muestra que los desarrollos tecnológicos que permiten mejoras en la eficiencia y el cambio 
hacia fuentes de energía bajas en emisiones no bastan. Por tanto, una transición muy masiva en consumo de materiales en todo el mundo puede incluso, temporalmente, disparar las emisiones.

- Se confía en poder hacer una transición del vehículo ligero de combustión al vehículo eléctrico, en tanto que para maquinaria pesada se reconoce que no hay aún tecnología apropiada (de ahí la cuestionable apuesta por el hidrógeno), y se debe investigar más. Se menciona explícitamente el riesgo de quedarse sin materiales críticos para baterías, pero se fía todo al reciclaje de materiales.

- El calentamiento global asociado a los distintos escenarios de emisiones publicados oscila entre menos de $1,5^{\circ} \mathrm{C}$ y más de $5^{\circ} \mathrm{C}$ para 2100 en comparación con los niveles preindustriales. Los escenarios de referencia sin nuevas políticas climáticas conducen a un calentamiento global medio de entre $3,3^{\circ} \mathrm{C}$ y $5,4^{\circ} \mathrm{C}$. La temperatura media de la Tierra es de cerca de 15 grados centígrados. Aumentar solo dos grados sería como aumentar casi cinco grados en un cuerpo humano. Quizá esta comparación ayude a entender el porqué de tanta preocupación con los famosos dos grados. La estabilidad climática sería imposible y el riesgo para la vida enorme. El problema es que la trayectoria actual no solo va directa a sobrepasar esos dos grados, sino que desatará aún más los temidos mecanismos de realimentación, que de no accionar sin dilación los frenos de emergencia del sistema, nos llevarían a un cambio climático ya absolutamente desbocado. Pese a esto no hay que paralizarse, hay posibilidades de evitar los peores escenarios, aunque hay que actuar coordinadamente. Ya.

- "No es incompatible luchar contra la pobreza energética y el cambio climático. Esto es así porque los grandes emisores son los más ricos: el 10\% más rico emite diez veces más que el 10\% más pobre. Por eso aumentar el consumo de los más pobres hasta niveles básicos de subsistencia no aumentaría mucho las emisiones".

- Destaca también la expansión de algunas actividades económicas intensivas en emisiones, por ejemplo "la aviación aumentando un $28,5 \%$ de 2010 a 2020". Pese a ello, a estas alturas, el Gobierno español está dando millonadas alegremente para las ampliaciones de los aeropuertos de Barajas y el Prat. Si se siguen los sucesivos informes que irá haciendo públicos el IPCC en los próximos meses, estos proyectos deberán verse como el absoluto sinsentido que son, salvo para los que se lucran con ellos. Evitar estas ampliaciones sería un buen punto de inflexión positivo, que podría significar un cambio de dinámica. 
- Reconocimiento de que hay un problema irresoluto con los plásticos.

- Se acepta implícitamente que los escenarios de mitigación suponen pérdidas del PIB. En el fondo, se admite lo que decía la propia Agencia Europea del Medio Ambiente: la preservación medioambiental no es compatible con el crecimiento económico. De hecho, el informe apunta: "En los escenarios que contemplan una reducción de la demanda energética, los retos de la mitigación se reducen significativamente, con una menor dependencia de la eliminación de $\mathrm{CO}_{2}(\mathrm{CDR})$, menor presión sobre la tierra y menores precios del carbono. Estos escenarios no suponen una disminución del bienestar, pero sí una provisión de mejores servicios.’ Esto es literalmente un escenario de adaptación al decrecimiento.

En cuanto a las medidas y pasos a dar, el informe destaca que no hay un mecanismo único de políticas o sistemas de gobernanza que por sí solos puedan acelerar la transición necesaria. Haría falta una combinación de éstas que será diferente en cada contexto.

Ejemplos de mecanismos serían los legislativos, que puede incentivar la mitigación proporcionando señales claras a los distintos actores, mediante el establecimiento de objetivos; o la creación de instituciones y mecanismos de mercado, como poner precio al carbono siempre que se tenga en cuenta la justicia social. Otros factores que pueden ayudar serían los movimientos sociales climáticos-el IPCC está reconociendo la labor de las huelgas por el clima-, que ayudan a provocar el aumento de otro de los factores clave: un alto porcentaje de gente comprometida. También se incide en que las medidas para conseguir reducciones tienen que ser cambios de comportamiento social: menos transporte, relocalización del trabajo, dieta más vegetariana, etc.

Y aquí está una de las claves, no se menciona claramente el imprescindible cambio radical en un sistema económico cuyo funcionamiento perverso de acumulación y reproducción del capital a perpetuidad nos ha llevado al punto crítico actual. Punto en el cual ya es difícil ocultar la evidencia de que otro punto, el de no retorno, está, como mínimo, muy cerca. Tal y como le ha ocurrido ya al Amazonas, que emite más carbono del que absorbe, o a Groenlandia, que ha batido récords de temperatura y de verter agua dulce y fría al océano, aumentando el riesgo de ralentización y colapso de la corriente termohalina, la cinta transportadora, vital para la estabilidad de nuestro sistema climático. $\mathrm{Y}$ cuyo colapso tendría consecuencias incalculables.

Ahí podemos entrever el pequeño/gran fallo del organismo, que por definición parece difícil de resolver y que hará que estas filtraciones 
se sigan produciendo: el IPCC funciona buscando consensos amplios, lo que dificulta que los posicionamientos más atrevidos sean tenidos en cuenta en el informe final, y no hablamos de científicos radicales solitarios: además de la Agencia Europea del Medio Ambiente, Nature, una de las revistas académicas más prestigiosas del mundo ya ha publicado estudios evidenciando que la única "solución" tanto para la transición energética como para la emergencia climática pasa por asumir que seguir creciendo sin causar más daño es obviamente imposible, y en consecuencia hay que planificar una estabilización y/o un decrecimiento de la esfera material. Repartir para vivir bien, pero dentro de los límites.

Recientemente, el barómetro de consumo responsable francés ha demostrado que la opinión pública está más abierta de lo que muchos quieren hacer creer. E1 52\% de los franceses creen que hay que abandonar por completo el modelo basado en el mito del crecimiento infinito. Así que la esperanza de que se trabaje en la línea de aceptar el problema y al mismo tiempo trabajar para remediarlo mundialmente de la manera más justa posible aumenta, a medida que decrecen nuestras posibilidades de seguir creciendo sin estrellarnos en el intento.

\title{
EL IPCC ADVIERTE DE QUE EL CAPITALISMO ES INSOSTENIBLE
}

\author{
Juan Bordera \\ Fernando Valladares ${ }^{\mathrm{b}}$ \\ Antonio Turiel \\ Ferran Puig Vilar \\ Fernando Prieto ${ }^{\mathrm{e}}$ \\ Tim Hewlett ${ }^{f}$
}

\footnotetext{
a Periodista, guionista y activista en Extinction Rebellion España y València en Transició.

b Doctor en Ciencias Biológicas, profesor de investigación en el CSIC y Premio Jaume I de Protección del Medio Ambiente.

${ }^{c}$ Doctor en Física Teórica, licenciado en Matemáticas, investigador científico en el CSIC y experto en energía. Es autor del reciente ensayo Petrocalipsis

${ }^{d}$ Ingeniero superior de Telecomunicaciones, periodista especializado en la crisis climática.

' Doctor en Ecología y director del Observatorio de la Sostenibilidad.

${ }^{\mathrm{f}}$ Doctor en Astrofísica y miembro del colectivo Scientist Rebellion.
} 
E1 segundo borrador del Grupo III del IPCC, el encargado de las propuestas de mitigación, afirma que hay que apartarse del capitalismo actual para no traspasar los límites planetarios. Confirma además lo que ya se adelantó en el artículo publicado en CTXT el pasado 7 de agosto: "Las emisiones de gases de efecto invernadero (GHG) deben tocar techo en como mucho cuatro años". El documento reconoce también que hay muy pocas posibilidades de seguir creciendo.

Los científicos y periodistas firmantes hemos analizado una nueva parte del Sexto Informe, filtrada por la misma fuente: el colectivo de científicos Scientist Rebellion y Extinction Rebellion España. En este apartado se pueden ver claramente las divergencias existentes en la comunidad científica con respecto a las medidas necesarias para realizar una transición efectiva y justa. Entre las habituales posiciones más tímidas, por fortuna, empiezan a asomar demandas que hace no mucho habría sido impensable que aparecieran.

Antes de entrar en el análisis, es preciso un poco de contexto: en 1990, el Primer Informe del IPCC todavía recogía que "el aumento observado [en la temperatura] podría deberse en gran medida a la variabilidad natural". Ese debate fue cerrándose en los siguientes informes. Pero si pervivía alguna duda, el análisis del Grupo I del Sexto Informe -ya oficial- ha despejado cualquier incertidumbre. Elimina así cualquier posibilidad de réplica por parte de un negacionismo climático, ampliamente regado de dinero por los que más tenían que perder: los lobbies de los combustibles fósiles. La primera pregunta para resolver un misterio suele ser el clásico Cui Bono (¿Quién se beneficia?).

El interrogante que subyace ahora guarda relación: ¿cómo hacemos para que la inevitable transición sea percibida como un beneficio y no como una renuncia? No hay otra posibilidad que renunciar al crecimiento indefinido, y el informe filtrado lo menciona. La transición ha de tener en cuenta las diferencias culturales e históricas de emisiones entre países, las diferencias entre el mundo rural y el urbano para no beneficiar a uno sobre otro, y sobre todo las tremendas y crecientes desigualdades económicas entre los cada vez más pobres y los cada vez más obscenamente ricos. $\mathrm{O}$ se atajan estas tres dicotomías, o la transición tendrá más enemigos que apoyos y se saboteará a sí misma. Textualmente el borrador dice: "Lecciones de la economía experimental muestran que la gente puede no aceptar medidas que se consideran injustas incluso si el coste de no aceptarlas es mayor".

Aun siquiera logrando cambiar de rumbo, los científicos advierten: "Las transiciones no suelen ser suaves y graduales. Pueden ser repen- 
tinas y perturbadoras". También señalan que "el ritmo de la transición puede verse obstaculizado por el bloqueo ejercido por el capital, las instituciones y las normas sociales existentes", enfatizando la importancia de las inercias. Y sobre ellas añaden: "La centralidad de la energía fósil en el desarrollo económico de los últimos doscientos años plantea cuestiones obvias sobre la posibilidad de la descarbonización”.

Las políticas favorables a las empresas de combustibles fósiles han extraído la riqueza común -nuestro aire, bosques, tierra...- y la han puesto en manos de una pequeña minoría. Por tanto, las políticas verdes tienen que ser obligatoriamente redistributivas en una época en la que la desigualdad se está disparando. Una de las medidas propuestas para reducir la regresividad de los precios del carbono es la redistribución de los ingresos fiscales para favorecer a las rentas bajas y medias. Pero, como recuerda el antropólogo Jason Hickel: todo lo que no sea un tope a la extracción de combustibles fósiles, con objetivos anuales decrecientes que reduzcan la industria a cero, será solo lavarse las manos.

Y llegamos a uno de los párrafos definitorios del informe: "Algunos científicos subrayan que el cambio climático está causado por el desarrollo industrial, y más concretamente, por el carácter del desarrollo social y económico producido por la naturaleza de la sociedad capitalista, que, por tanto, consideran insostenible en última instancia". Aunque muchos lo han dicho antes, no creemos haber leído jamás nada tan clarificador en el informe climático más importante del mundo, que añade: "Las emisiones actuales son incompatibles con el Acuerdo de París por lo que es absolutamente obligatorio reducirlas de una forma inmediata y contundente".

Gráfica 1

Emisiones de gases efecto invernadero: tendencias y proyecciones 2000-2050

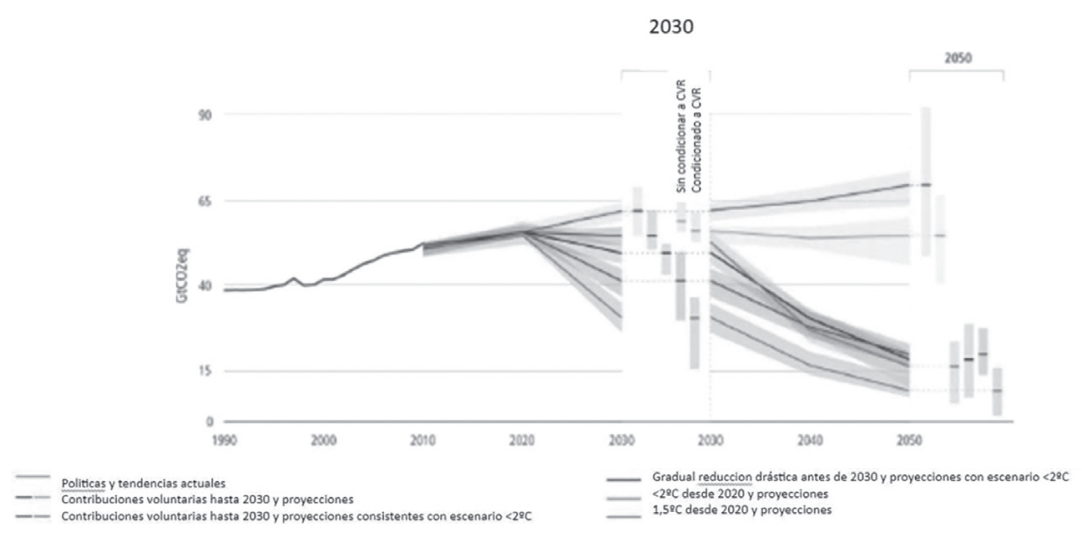

Revista de Economía Institucional, vol. 24 , N. ${ }^{\circ} 46$, Primer semestre/2022, pp. $237-247$ ISSN OI $24-5996 / \mathrm{E}-\mathrm{ISSN} 2346-2450$ 


\section{Diferentes escenarios de Reducción De EMisiones}

Estas metas, que implican un decrecimiento drástico de las emisiones y, por tanto, a corto plazo también de la producción de energía y del uso de materiales, son imposibles de lograr con el modelo actual. Además, el Grupo III vincula la reducción de las emisiones con el cumplimiento de los 17 Objetivos de Desarrollo Sostenible, acordados en 2015 por los países miembros de Naciones Unidas para su cumplimiento en 2030. A pesar de las contradicciones existentes entre los 17 ODS, entre ellos encontramos objetivos incuestionables como la reducción de la desigualdad y la protección de la biodiversidad, entremezclados con uno más polémico, dentro del propio informe: promover el crecimiento económico sostenible.

En el IPCC es costumbre no esconder el debate científico, y si en 1990 este giraba aún sobre las causas del cambio climático, tras 30 infructuosos años, podemos observar que la discusión oscila ahora entre aquellas posiciones que aún creen que se puede seguir creciendo y reducir las emisiones al ritmo necesario, y quienes vemos esto como otro tipo de negacionismo, más sutil, pero que en el fondo beneficia $\mathrm{y}$ es defendido por los mismos que antaño cuestionaban el origen del calentamiento global.

El informe del IPCC asume que "los objetivos de mitigación y desarrollo no pueden alcanzarse mediante cambios incrementales". Obcecarse en el crecimiento exige desarrollar enormemente tecnologías que puedan reducir las concentraciones de gases de efecto invernadero en la atmósfera, pero esas tecnologías de CCS (Captura y Secuestro de Carbono) no se están materializando como se preveía.

Con los sumideros de carbono de los ecosistemas en claro declive y las retroalimentaciones climáticas que se están desencadenando -lo que lleva a la Tierra a sobrepasar varios puntos de no retorno, como reconoce ya una amplia mayoría, y de ahí a un estado más caliente e inestable-, la única forma conocida de evitar el colapso climático es apartarse del modelo de crecimiento perpetuo.

$\mathrm{El}$ informe destaca que en cooperación internacional se ha identificado una "hipocresía organizada" en la que los acuerdos y afirmaciones no concuerdan con las acciones, lo que supone una de las barreras más importantes para la mitigación.

E1 IPCC también apela a no olvidar las lecciones no puestas en práctica de la covid-19. Lecciones que deberían servir para no cometer los mismos errores con el cambio climático, ya que las analogías son claras y directas. Los costes de prevención y acciones preparativas 
son mínimos comparados con los costes de los impactos causados. Retrasar las medidas tendrá costes crecientes muy difíciles de asumir.

\section{Gráfica 2}

Posibilidad de impactos negativos con acciones inmediatas y retrasadas en el tiempo

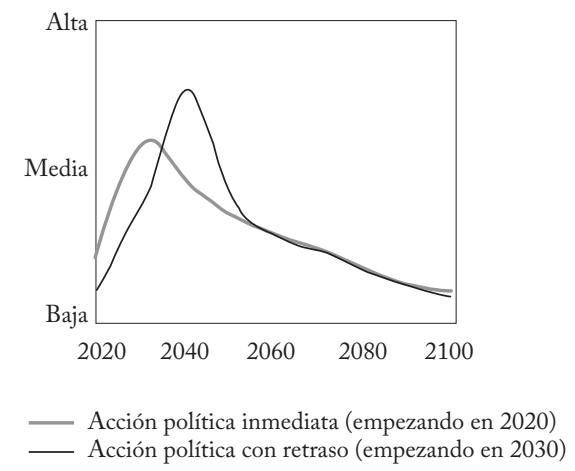

Escenarios medios compatibles con subidas de $1,5^{\circ} \mathrm{C}$ y $2^{\circ} \mathrm{C}$ a final de siglo.

Fuente: borrador Grupo III. Agosto 2021. Reelaborado por CTXT/Observatorio Sostenibilidad.

\section{DE NO ACTUAR PRONTO LOS RETOS AUMENTARÁN DE FORMA NO LINEAL Y CON CONSECUENCIAS IMPREVISTAS}

Vistas las cada vez más evidentes contradicciones del concepto de desarrollo sostenible, hablar de cualquier forma de desarrollo solo será posible si se deja de lado al PIB como medidor de riqueza, y se cambia a un modelo económico no tan basado en la competición. E1 único desarrollo sostenible es horizontal, no vertical. Es decir, reducir la desigualdad.

Es evidente que, o existe la percepción de que una gran mayoría nos 'beneficiamos', o no habrá solución. Por ello se ha de explicar bien la enorme magnitud del problema para que las medidas puedan ser comprendidas y ciertas renuncias puedan ser entendidas como beneficios, si tenemos en cuenta que la alternativa es cambiar la estabilidad climática para siempre y agravar los conflictos por los recursos.

La competición ayudó a desarrollar la evolución de las especies, pero, tal y como demostró la genial microbióloga Lynn Margulis, la cooperación es la clave que explica los grandes saltos ervolutivos. Ahora nos encontramos ante un precipicio dibujado por la intersección de las crisis ecológica y energética. Podemos tener vidas buenas con menos energía disponible (y a la vez tendremos menos carga laboral), pero el capitalismo no podrá sostenerse con menos energía sin finalizar su mutación a una especie de tecnofeudalismo. Solo si cooperamos, si 
entendemos que compartimos tantas cosas -entre ellas una atmósfera que no sabe qué es eso de las fronteras- podremos reaccionar y saltar lo suficiente como para evitar la caída. 